\title{
Theory of excitons in carbon nanotubes
}

\author{
Tsuneya Ando ${ }^{1, *}$, Seiji Uryu² \\ ${ }^{1}$ Department of Physics, Tokyo Institute of Technology, 2-12-1 Ookayama, Meguro-ku, Tokyo 152-8551, Japan \\ ${ }^{2}$ Department of Physics and Electronics, Osaka Prefecture University, 1-1 Gakuen-cho, Sakai, Osaka 599-8531, Japan
}

Received XXXX, revised XXXX, accepted XXXX

Published online XXXX

PACS 78.67.Ch, 73.22.Lp

${ }^{*}$ Corresponding author: e-mail ando@phys.titech.ac.jp, Phone +81-3-5734-2072, Fax +81-3-5734-2739

A brief review is given on the electronic and optical properties of carbon nanotubes. The topics include an effectivemass description of the electronic states, optical absorption and excitons, two-photon absorption, excitons in metallic nanotubes, their fine structure and the Aharonov-Bohm effect, and exciton absorption for perpendicular polarization.

Copyright line will be provided by the publisher

1 Introduction Carbon nanotubes $(\mathrm{CN})$ are either a metal or semiconductor, depending on their diameters and helical arrangement. This condition can be obtained based on the band structure of a two-dimensional (2D) graphite sheet and periodic boundary conditions along the circumference direction. This result was predicted first by means of a tight-binding model and also by a k.p method or an effective-mass approximation $[1,2]$. The cylindrical shape leads to a strong Aharonov-Bohm (AB) effect in the band structure due to a magnetic field parallel to the axis [3]. The purpose of this paper is to give a brief review of electronic and optical properties, predicted in the $\mathbf{k} \cdot \mathbf{p}$ scheme. The topics include roles of excitons in optical absorption, twophoton absorption, excitons in metallic nanotubes, their fine structure and the Aharonov-Bohm effect, and exciton absorption for perpendicular polarization.

2 Effective-mass description A mono-layer graphite (graphene) sheet is a zero-gap semiconductor in which the conduction and valence bands consist of $\pi$ states that cross at the $\mathrm{K}$ and $\mathrm{K}$ ' points of the Brillouin zone [4]. The structure is shown in Fig. 1 together with the first Brillouin zone and coordinate systems to be used in the following. Electronic states near a $\mathrm{K}$ point are described by the $\mathbf{k} \cdot \mathbf{p}$ equation $[1,2,5]$ :

$$
\gamma(\boldsymbol{\sigma} \cdot \hat{\mathbf{k}}) \mathbf{F}(\mathbf{r})=\varepsilon \mathbf{F}(\mathbf{r}), \quad \mathbf{F}(\mathbf{r})=\left(\begin{array}{c}
F_{A}(\mathbf{r}) \\
F_{B}(\mathbf{r})
\end{array}\right),
$$

where $\gamma$ is the band parameter, $\hat{\mathbf{k}}=\left(\hat{k}_{x}, \hat{k}_{y}\right)=-i \nabla$ is a wave-vector operator, $\varepsilon$ is the energy, and $\sigma_{x}$ and $\sigma_{y}$ are the
Pauli spin matrices. Two components of the wave function $\mathbf{F}(\mathbf{r})$ correspond to the amplitude at $\mathrm{A}$ and $\mathrm{B}$ sites in a unit cell. Equation (1) has the form of Weyl's equation for neutrinos. If we use a nearest-neighbor tight-binding model with hopping integral $-\gamma_{0}$, the band parameter becomes $\gamma=(\sqrt{3} / 2) a \gamma_{0}$, where $a$ is the lattice constant.

The structure of a nanotube is specified by a chiral vector $\mathbf{L}$ corresponding to the circumference as shown in Fig. 1 . In the following we shall choose the $x$ axis in the circumference direction and the $y$ axis in the axis direction, i.e., $\mathbf{L}=(L, 0)$ with $L=|\mathbf{L}|$. The angle $\eta$ between $\mathbf{L}$ and the horizontal axis is called the chiral angle.

The electronic states can be obtained by imposing the periodic boundary condition in the circumference direction, $\psi(\mathbf{r}+\mathbf{L})=\psi(\mathbf{r})$, except in extremely thin tubes. The Bloch functions at a $\mathrm{K}$ point change their phase by $\exp (i \mathbf{K} \cdot \mathbf{L})=\exp (2 \pi i \nu / 3)$, where $\nu=0$ or \pm 1 , determined by $\mathbf{L}$. Because $\psi(\mathbf{r})$ is written as a product of the Bloch function and the envelope function, i.e., the neutrino wave function $\mathbf{F}(\mathbf{r})$, this phase change should be canceled by that of the envelope functions and the boundary conditions are given by $\mathbf{F}(\mathbf{r}+\mathbf{L})=\mathbf{F}(\mathbf{r}) \exp (-2 \pi i \nu / 3)$.

A nonzero curvature causes a shift in the origin of $\hat{k}_{x}$ and $\hat{k}_{y}$ in the $\mathbf{k} \cdot \mathbf{p}$ Hamiltonian. The shift in the $y$ direction is irrelevant and that in the $x$ direction can be replaced by an effective flux $\phi_{c}$. The flux was estimated as $[2,6]$

$$
\frac{\phi_{c}}{\phi_{0}}=\frac{2 \pi}{4 \sqrt{3}} \frac{a}{L} p \cos 3 \eta
$$



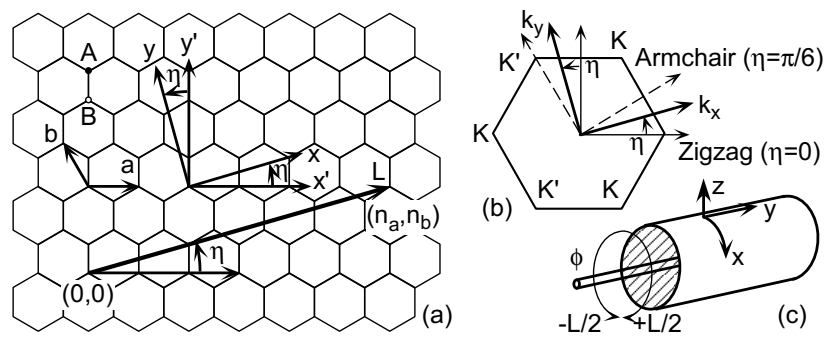

Figure 1 (a) Lattice structure of a two-dimensional graphite (graphene) sheet. The coordinates $\left(x^{\prime}, y^{\prime}\right)$ are fixed on the graphite sheet and $(x, y)$ are chosen in such a way that $x$ is along the circumference and $y$ is along the axis. (b) The first Brillouin

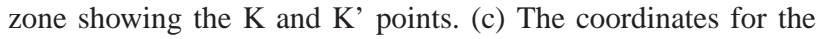
nanotube. An Aharonov-Bohm flux $\phi$ is applied along the $y$ axis direction of the nanotube.

with $\phi_{0}=c h / e$ being the flux quantum, $p=1-(3 / 8) \gamma^{\prime} / \gamma$, $\gamma=-(\sqrt{3} / 2) V_{p p}^{\pi} a$, and $\gamma^{\prime}=-(\sqrt{3} / 2)\left(V_{p p}^{\sigma}-V_{p p}^{\pi}\right) a$, where $V_{p p}^{\pi}\left(=-\gamma_{0}\right)$ and $V_{p p}^{\sigma}$ are the conventional tightbinding parameters for neighboring $p$ orbitals [6]. The curvature effect is largest in zigzag nanotubes with $\eta=0$ and absent in armchair nanotubes with $\eta=\pi / 6$. The above flux is different from that given in Ref. [7] in which $V_{p p}^{\sigma}$ was neglected.

The presence of a lattice distortion $\mathbf{u}=\left(u_{x}, u_{y}, u_{z}\right)$ also causes an effective flux. It is estimated as [8]

$$
\frac{\phi_{s}}{\phi_{0}}=\frac{L g_{2}}{2 \pi \gamma}\left[\left(u_{x x}-u_{y y}\right) \cos 3 \eta-2 u_{x y} \sin 3 \eta\right]
$$

where $u_{\mu \nu}(\mu, \nu=x, y)$ denotes the strain tensor given by $u_{x x}=\left(\partial u_{x} / \partial x\right)+\left(2 \pi u_{z} / L\right), u_{y y}=\partial u_{y} / \partial y, 2 u_{x y}=$ $\left(\partial u_{x} / \partial y\right)+\left(\partial u_{y} / \partial x\right)$, and $g_{2}$ is the interaction energy given by $g_{2}=(\alpha / 2) \gamma_{0}$ with $\alpha \sim 1$ [8]. This shows that twist and stretch deformation give rise to nonzero flux in armchair and zigzag nanotubes, respectively. The coupling constant $g_{2}$ is about a factor of three smaller than that discussed using a tight-binding model $[9,10]$.

In summary, the electronic states in nanotubes can be specified by a single parameter $\varphi_{e}$ defined by $\varphi_{e}=-(\nu / 3)$ $+\varphi_{c}+\varphi_{s}$, with $\varphi_{c}=\phi_{c} / \phi_{0}$ and $\varphi_{s}=\phi_{s} / \phi_{0}$, and energy levels for the K point are obtained by putting $k_{x}=\kappa_{\varphi_{e}}(n)$ with $\kappa_{\varphi_{e}}(n)=(2 \pi / L)\left(n+\varphi_{e}\right)$ and $k_{y}=k$ in the above $\mathbf{k}$. p equation as $\varepsilon_{\varphi_{e}}^{( \pm)}(n, k)= \pm \gamma \sqrt{\kappa_{\varphi_{e}}(n)^{2}+k^{2}}$, where $n$ is an integer $(n=0, \pm 1, \cdots)$ and the upper $(+)$ and lower $(-)$ signs represent the conduction and valence bands, respectively. For the K' point, the Schrödinger equation is given by replacing $\sigma$ with complex conjugate $\sigma^{*}$ and the boundary condition gives an effective flux $-\varphi_{e}$ leading to $k_{x}=\kappa_{-\varphi_{e}}(n)$. When $\phi_{c}=\phi_{s}=0$, a nanotube becomes metallic for $\nu=0$ and semiconducting with gap $\varepsilon_{G}=4 \pi \gamma / 3 L$ for $\nu= \pm 1$.

When a magnetic field is applied parallel to the axis, i.e., in the presence of a magnetic flux $\phi$ passing through
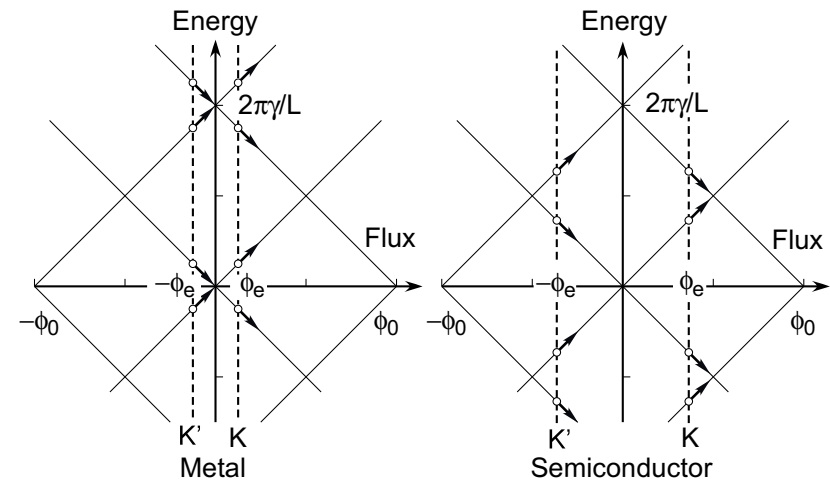

Figure 2 The band edges $\pm(2 \pi \gamma / L)\left|n-\left(\phi / \phi_{0}\right)\right|$ as a function of $\phi$ for metallic and semiconducting tubes. In the presence of an effective flux $\phi_{e}$ due to curvature and/or strain, we have $\phi=\phi_{e}$ for the $\mathrm{K}$ point and $\phi=-\phi_{e}$ for the $\mathrm{K}^{\prime}$ point. An $\mathrm{AB}$ magnetic flux shifts $\phi$ in the positive direction as shown by arrows.

the cross section, the $\mathrm{AB}$ flux leads to the change in the boundary condition $\psi(\mathbf{r}+\mathbf{L})=\psi(\mathbf{r}) \exp (+2 \pi i \varphi)$, where $\varphi=\phi / \phi_{0}$. Consequently, $\kappa_{\varphi_{e}}(n)$ is replaced with $\kappa_{\varphi+\varphi_{e}}(n)$ for the K point and $\kappa_{-\varphi_{e}}(n)$ with $\kappa_{\varphi-\varphi_{e}}(n)$ for the K' point. The energy bands are given by $\varepsilon_{\varphi+\varphi_{e}}^{( \pm)}(n, k)$ and $\varepsilon_{\varphi-\varphi_{e}}^{( \pm)}$ $(n, k)$ for the $\mathrm{K}$ and $\mathrm{K}$ ' points, respectively. Figure 2 shows a schematic illustration of the band edges $\varepsilon_{\varphi \pm \varphi_{e}}^{( \pm)}(n, 0)$. The gap exhibits an oscillation between 0 and $2 \pi \gamma / L$ with period $\phi_{0}$ with the change in the $\mathrm{AB}$ flux $\phi[1]$. This giant $\mathrm{AB}$ effect on the band gap is a unique property of nanotubes.

3 Excitons A carbon nanotube has characteristic optical properties. In fact, the absorption of light polarized perpendicular to the axis is suppressed because of a strong depolarization effect [11,12], and the Coulomb interaction plays a crucial role. Interaction effects on the band structure were evaluated within a conventional screened HartreeFock approximation and were shown to enhance the band gap [13]. Further, it was shown that the exciton effect is extremely important because of the one-dimensional nature of the nanotube [13].

Within the effective-mass approximation, the strength of electron-electron interaction is characterized by the dimensionless parameter $\left(e^{2} / \kappa L\right)(2 \pi \gamma / L)^{-1}$ independent of $L$, where $\kappa$ is a static dielectric constant describing the effects of the polarization of electrons of core states, $\sigma$ bands, $\pi$ bands away from the $\mathrm{K}$ and $\mathrm{K}^{\prime}$ points, and the surrounding material. In bulk graphite we have $\kappa \approx 2.4$ [14]. Although the exact value of $\kappa$ is unknown in nanotubes, the interaction parameter is expected to lie in the range $0.1 \lesssim\left(e^{2} / \kappa L\right)(2 \pi \gamma / L)^{-1} \lesssim 0.2$.

A later calculation of quasi-particle energies using a full dynamical random-phase approximation $[15,16]$, which is often called the GW approximation [17], showed that the screened Hartree-Fock approximation works sufficiently well in semiconducting nanotubes and in metallic nanotubes apart 


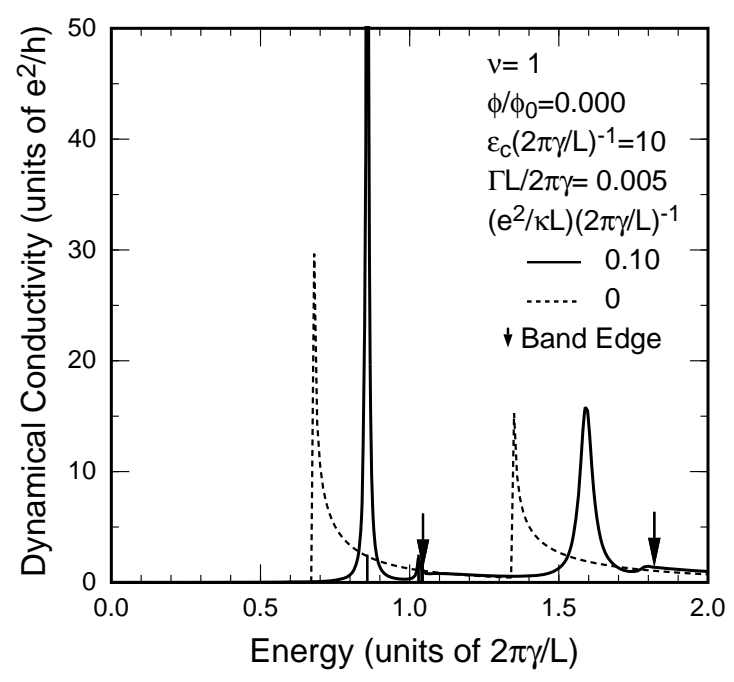

Figure 3 Calculated optical absorption spectrum of a semiconducting nanotube. The solid and dotted lines represent spectra in the presence and absence of interaction, respectively. The band edges are indicated by vertical arrows. After Ref. [18].

from bands with a linear dispersion. It was demonstrated that the self-energy shift depends on the cutoff energy, leading to an extra logarithmic dependence on the diameter. The cutoff energy $\varepsilon_{c}$ is of the order of the half of the $\pi$ band width $\sim 3 \gamma_{0}$. This leads to $\varepsilon_{c}(2 \pi \gamma / L)^{-1} \sim(\sqrt{3} / \pi)(L / a)$, i.e., $\varepsilon_{c}(2 \pi \gamma / L)^{-1} \sim 10$ for typical CN's with diameter $\sim 1.4 \mathrm{~nm}$. The energy bands and excitons were then calculated carefully including this cutoff dependence [18].

A dominant feature is the enhancement of the band gaps with the increase of the interaction strength. The binding energy of excitons increases with the interaction but remains smaller than the band-gap enhancement. As a result, the absorption energies become higher than the band gap without interaction. Further, interaction effects on the band gap and the exciton binding energy are larger for the second band than for the first band. Another important feature is that the ratio of the excitation energies of the second gap and that of the first gap is essentially independent of the cutoff energy and therefore of the diameter. Further, the ratio for the exciton energies decreases from two in the absence of interaction to about 1.8 in the presence of the interaction, in agreement with experiments.

Some examples of the absorption spectra are shown in Fig. 3 for $\varepsilon_{c}(2 \pi \gamma / L)^{-1}=10$ and $\left(e^{2} / \kappa L\right)(2 \pi \gamma / L)^{-1}$ $=0.1$. Most of the absorption intensity is transferred to the exciton ground state from the inter-band continuum for both the first and second gaps. In the present scheme the relevant parameter is $\gamma$ or $\gamma_{0}$. The calculated absorption energies are in agreement with some of existing experiments [19-21] for $\gamma_{0}=2.7 \mathrm{eV}$.

Quasi-particle spectra of nanotubes were calculated using a first-principles GW method [22], and calculations were performed also for optical absorption spectra with the inclusion of excitonic final state interactions [23-26]. There have also been some reports on a phenomenological description of excitons [27,28].

4 Two-photon absorption The information on excited exciton states is known to be accessed by two-photon absorption process [29-35]. It is straight-forward to calculate two-photon spectra associated with exciton excited states for the polarization parallel to the tube axis [36]. Figure 4 shows an example of the results, which show the appearance of a prominent peak corresponding to the second lowest exciton with even parity and another weak peak associated with the fourth lowest exciton. The difference between the first and second lowest exciton energies is approximately 0.15 and 0.22 in units of $2 \pi \gamma / L$ for $\left(e^{2} / \kappa L\right)$ $\times(2 \pi \gamma / L)^{-1}=0.1$ and 0.2 , respectively.

In Fig. 5, excitation energies are plotted as a function of the circumference length. Solid and dotted lines denote calculated energies of the two lowest excitons observable in one- and two-photon absorption, respectively, and dashed lines indicate band edges. Symbols are experimental results $[34,35]$ where open and filled symbols indicate excitons for one- and two-photon transitions, respectively.

The energy of the lowest exciton is weakly dependent on the interaction strength $\left(e^{2} / \kappa L\right) \times(2 \pi \gamma / L)^{-1}$. Therefore, the band parameter is uniquely determined as approximately $\gamma_{0} \approx 2.7$ [18]. Using the position of the second exciton, strongly dependent on the Coulomb interaction, we can place the interaction parameter in the range $0.1<$ $\left(e^{2} / \kappa L\right) \times(2 \pi \gamma / L)^{-1}<0.2$. More precise determination becomes possible when we include other effects such as a higher order $\mathbf{k} \cdot \mathbf{p}$ terms giving trigonal warping, curvature,

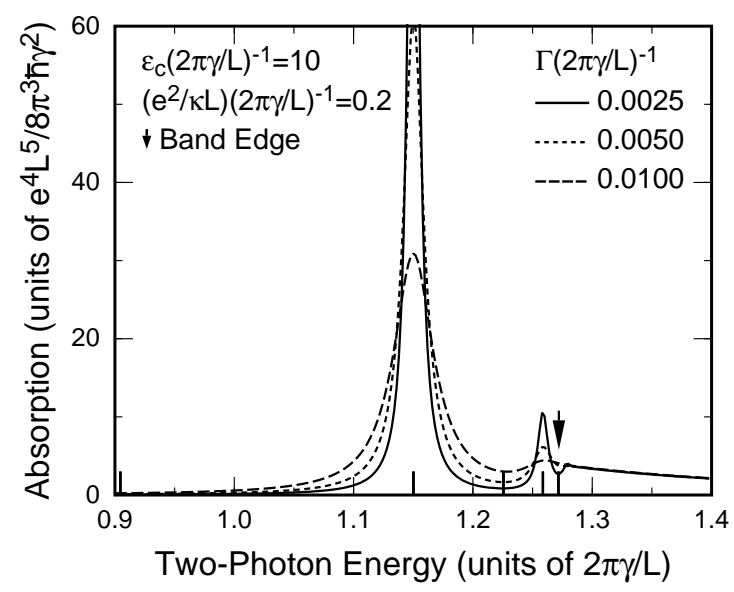

Figure 4 Calculated two-photon absorption coefficient for $\left(e^{2} / \kappa L\right)(2 \pi \gamma / L)^{-1}=0.2$. Phenomenological broadening $\Gamma$ $\times(2 \pi \gamma / L)^{-1}$ is introduced. The arrows indicate the band edge and the short vertical lines at the bottom denote exciton energies. After Ref. [36]. 


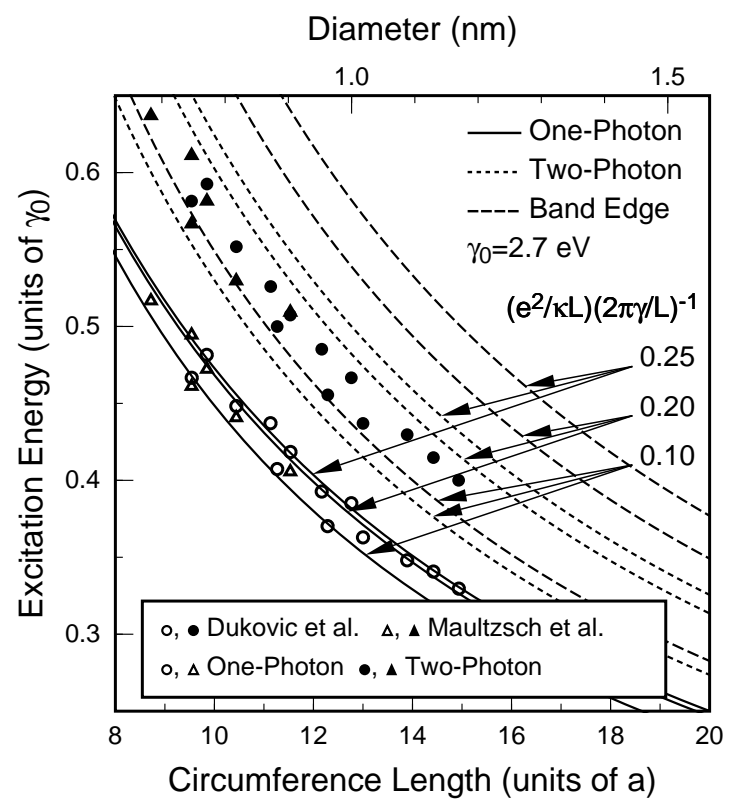

Figure 5 Calculated excitation energies as a function of the circumference length. Solid and dotted lines denote the energies for one- and two-photon absorption, respectively, and dashed lines are the band edges. Symbols denote experimental results $[34,35]$ where open and closed symbols indicate one- and two-photon transitions, respectively. After Ref. [36].

and lattice distortions [18], leading to a family effect observed experimentally $[21,37]$.

5 Excitons in metallic nanotubes In metallic nanotubes, photoluminescence measurement is not possible and various other methods such as optical absorption [19,20, 38], Raman scattering [39-41], and Rayleigh spectroscopy [42] have been used. These experiments gave rich information, including possible assignment of observed spectra to different diameters and chirality, peak splitting due to trigonal warping effect, etc. Because of strong screening exciton effects are usually not important in metallic systems. However, excitons can survive in nanotubes due to their strong binding and weaker screening.

Theoretically, excitons in metallic nanotubes were first studied in an effective-mass approximation [13]. A considerable enhancement of both optical gap and band gap due to $\mathrm{AB}$ flux was shown and the absence of excitonic instability was revealed for the linear bands in a limit of zero flux. Recently, exciton effects in metallic nanotubes were explicitly addressed [43], and first-principles calculations gave binding energies of excitons typically one-order-ofmagnitude smaller than those of semiconducting nanotubes $[23,44]$.

A systematic study of excitons in metallic tubes has also been performed within the $\mathbf{k} \cdot \mathbf{p}$ scheme [45]. Typical examples of optical absorption spectra for small magnetic

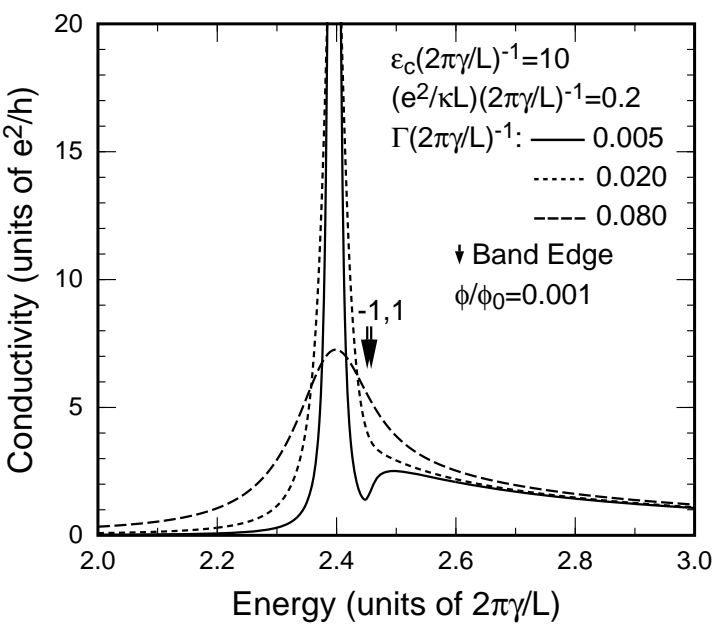

Figure 6 Dynamical conductivity for parallel polarization at $\varphi=0.001$. Arrows indicate edges of the bands for $n= \pm 1$ and numbers denote band index $n$. The phenomenological energy broadening $\Gamma(2 \pi \gamma / L)^{-1}=0.005$ (solid lines), 0.02 (dotted liens), and 0.08 (dashed lines) is used. After Ref. [45].

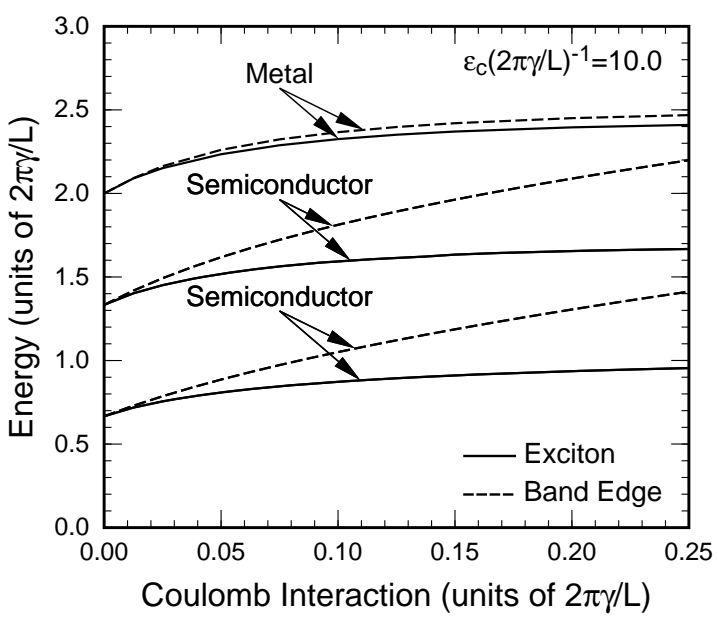

Figure 7 Coulomb-interaction dependence of the exciton energies (solid lines) and band edges (dashed lines) for parallel polarization for the bands with $n= \pm 1$ of metallic tubes and for the lowest and second lowest excitation of semiconducting tubes. After Ref. [45].

flux $\varphi=0.001$ are shown in Fig. 6. At around energy $2.4 \times(2 \pi \gamma / L)$, a sharp exciton peak appears. In the energy region higher than the band edges denoted by arrows, contributions from continuum states can be seen as a small and long tail. The binding energy approximately takes a value $\sim 0.05 \times(2 \pi \gamma / L)$, one-order-of-magnitude smaller than that for semiconducting nanotubes.

In Fig. 7, the result is compared with those of the first and second gaps in semiconducting nanotubes. The interaction dependence of the band edge of metallic tubes is 
weaker than that of semiconducting tubes. However, the small exciton binding-energy leads to the dependence of the exciton energy similar to those for semiconducting tubes.

6 Fine structure and Aharonov-Bohm effect Because of the electron spin and the presence of $\mathrm{K}$ and $\mathrm{K}$ ' points, there are 16 exciton states. They can be written for example as $\left|K K^{\prime}\right\rangle$, where an electron is in the conduction band at the $\mathrm{K}$ point and a hole in the valence band at the K' point. Similar definitions can be made for other combinations. First, by a short-range part of the Coulomb interaction giving rise to scattering of an electron between the $\mathrm{K}$ and $\mathrm{K}^{\prime}$ points, the excitons $|K K\rangle$ and $\left|K^{\prime} K^{\prime}\right\rangle$ are coupled to form a bonding state $\left|K K-K^{\prime} K^{\prime}(+)\right\rangle$ with a lower energy and a antibonding $\left|K K-K^{\prime} K^{\prime}(-)\right\rangle$ with a higher energy. Because of the momentum conservation, the $\left|K K^{\prime}\right\rangle$ and $\left|K^{\prime} K\right\rangle$ excitons remain decoupled by this interaction. The matrix element of the interaction is proportional to $\Omega_{0} w_{2}$, where $\Omega_{0}=\sqrt{3} a^{2} / 2$ is the area of a unit cell and $w_{2}$ is given by $w_{2} \approx \sum_{\mathbf{R}} V(\mathbf{R}) e^{i\left(\mathbf{K}-\mathbf{K}^{\prime}\right) \cdot \mathbf{R}}$ with $V(\mathbf{R})$ being the Coulomb potential between $\pi$ orbitals with lattice separation $\mathbf{R}$ [46].

These excitons split into spin-singlet and triplet due to the exchange interaction. Among them only the singlet $\left|K K-K^{\prime} K^{\prime}(+)\right\rangle$ exciton is optically allowed (bright) and all others do not contribute to absorption and emission (dark). The exchange interaction arising due to a short-range part of the Coulomb interaction within the $\mathrm{K}$ and $\mathrm{K}$ ' points is proportional to $\Omega_{0} w_{1}$ with $w_{1} \approx(1 / 2) \sum_{\mathbf{R}}[V(\mathbf{R})-$ $V(\mathbf{R}-\boldsymbol{\tau})]$, where $\boldsymbol{\tau}$ is a vector connecting nearest neighbor atoms shown in Fig. 1 [46]. When we assume the simple Coulomb form $V(\mathbf{R}) \propto e^{2} /|\mathbf{R}|$ for $\mathbf{R} \neq 0$, we have $w_{1} \approx(1 / 2)\left[V(0)-c_{1} V(a)\right]$ and $w_{2} \approx V(0)-c_{2} V(a)$, where $c_{1}$ and $c_{2}$ are constants given by $c_{1}=2.67 \cdots$ and $c_{2}=1.54 \cdots$ determined by the lattice structure alone. This shows that $w_{1} \lesssim w_{2} / 2$. If we use the Ohno potential $V(\mathbf{R})=U\left[\left(U|\mathbf{R}| / e^{2}\right)^{2}+1\right]^{-1 / 2}$ with $U \approx 11.3 \mathrm{eV}[47$, 48], we have $w_{1} / w_{2} \sim 0.17$.

The amount of the splitting and shift is of the order of $(a / L)^{2} w_{2}$ or $(a / L)^{2} w_{1}$ in contrast to $\propto a / L$ suggested in Ref. [49], and therefore becomes rapidly smaller with $L$. Figure 8 shows the exciton energy levels determined by $w_{1}$ and $w_{2}$ as a function of $w_{1} / w_{2}$. Except in the case of $w_{1}=0$, the triplet states are lower in energy and the bright exciton, i.e., singlet $\left|K K-K^{\prime} K^{\prime}(+)\right\rangle$, increases in the energy with the increase of $w_{1} / w_{2}$. Around at $w_{1} / w_{2}=0.25$ it becomes higher than the dark singlet exciton $\mid K K-K^{\prime} K^{\prime}$ $(-)\rangle$.

This exciton fine structure is strongly affected by an Aharonov-Bohm magnetic flux because of the splitting of the $\mathrm{K}$ and $\mathrm{K}$ ' points as shown in Fig. 2. This AharonovBohm splitting was observed in recent experiments [5055]. Figure 9 shows some examples of the energy levels of the singlet and triplet excitons as a function of the flux for the parameter $w_{2} / \gamma_{0}=1.5$ in nanotubes with circumference $L / a=20$ and for $w_{1} / w_{2}=0.4$. In this case

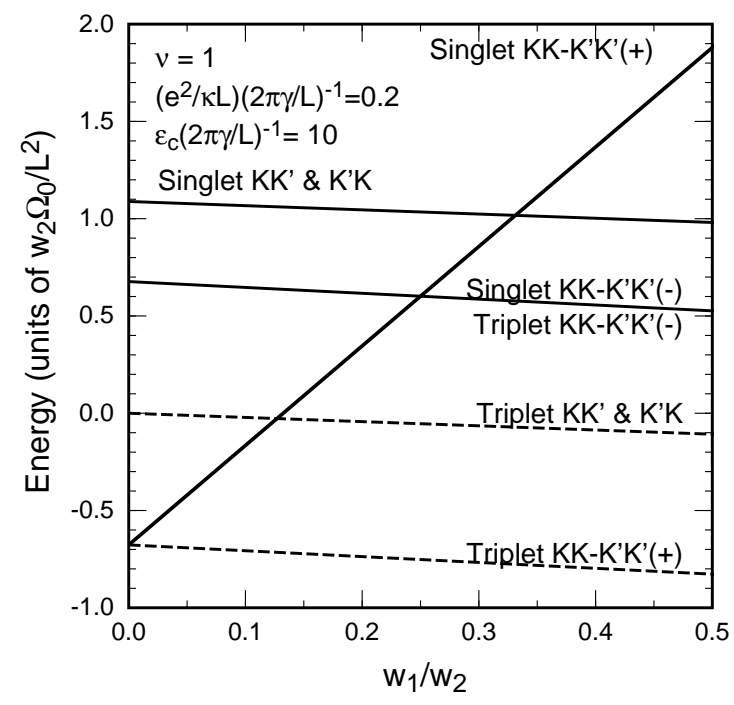

Figure 8 The energy levels of excitons as a function of $w_{1} / w_{2}$. The singlet and triplet states are degenerate for $\left|K K-K^{\prime} K^{\prime}(-)\right\rangle$. The crossing of singlet $\left|K K-K^{\prime} K^{\prime}(+)\right\rangle$ (bright) and $\left|K K-K^{\prime} K^{\prime}(-)\right\rangle$ (dark) occurs around at $w_{1} / w_{2}=$ 0.25. $\left(e^{2} / \kappa L\right) \times(2 \pi \gamma / L)^{-1}=0.2$. After Ref. [46].

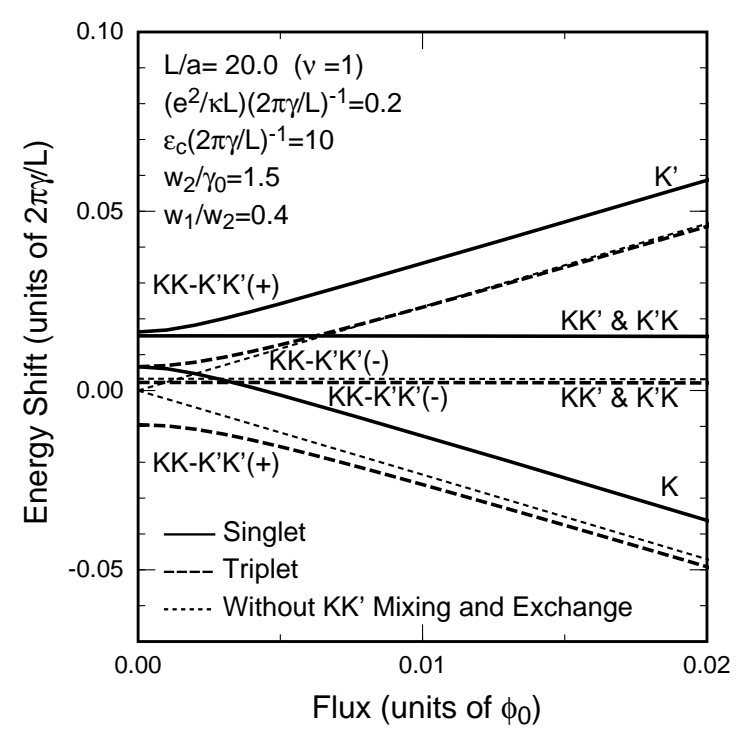

Figure 9 Calculated energy levels of the singlet and triplet excitons as a function of the flux for the parameter $w_{2} / \gamma_{0}=1.5$ in semiconducting nanotubes with $\nu=1$ and circumference $L / a=20 .\left(e^{2} / \kappa L\right)(2 \pi \gamma / L)^{-1}=0.2 . w_{1} / w_{2}=0.2$. After Ref. [46].

the highest-energy exciton is bright in the absence of flux. Mixing between the $\mathrm{K}$ and $\mathrm{K}$ ' points diminishes rapidly with the increase of the flux and excitons for the $\mathrm{K}$ and $\mathrm{K}$ ' points become independent of each other for a sufficiently large $\mathrm{AB}$ flux. On the other hand, $\left|K K^{\prime}\right\rangle$ and $\left|K^{\prime} K\right\rangle$ exci- 


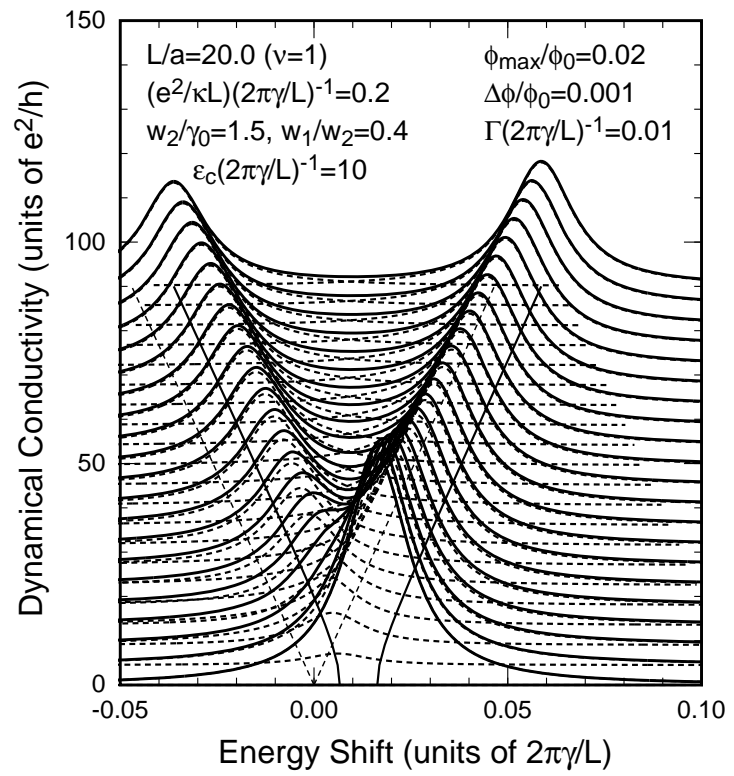

Figure 10 Calculated dynamical conductivity describing the absorption spectra. The solid lines represent the conductivity and the dotted lines contributions of two singlet KK-K' $\mathrm{K}^{\prime}$ excitons. $L / a=10 .\left(e^{2} / \kappa L\right)(2 \pi \gamma / L)^{-1}=0.2 . w_{1} / w_{2}=0.4$. After Ref. [46].

tons remain at the same energy because of the cancellation of flux effects between the conduction and valence bands.

Figure 10 shows some examples of the dynamical conductivity describing the absorption spectra for $w_{1} / w ? 2=$ 0.4. In the absence of flux, a single absorption peak appears only at the energy of the bonding combination of $|K K\rangle$ and $\left|K^{\prime} K^{\prime}\right\rangle$ excitons depending on the ratio $w_{1} / w_{2}$ as mentioned above. When $w_{1} / w_{2}>0.25$ as in the figure, the bright $\left|K K-K^{\prime} K^{\prime}(+)\right\rangle$ exciton lies higher in energy than the dark $\left|K K-K^{\prime} K^{\prime}(-)\right\rangle$ exciton and a new peak appears in the low energy side. When $w_{1} / w_{2}<0.25$, on the other hand, the bright $\left|K K-K^{\prime} K^{\prime}(+)\right\rangle$ exciton lies lower than the dark $\left|K K-K^{\prime} K^{\prime}(-)\right\rangle$ exciton and a new peak appears in the high energy side.

An important characteristic feature of the experimental results of the Aharonov-Bohm splitting [50-55] is that the splitting does not seem to become observable until the magnetic flux reaches a certain critical value and then starts to increase with the flux. This is consistent with the above result that two peaks become observable only when the Aharonov-Bohm splitting exceeds the mixing between the $|K K\rangle$ and $\left|K^{\prime} K^{\prime}\right\rangle$ exciton due to the short-range Coulomb interaction. The Aharonov-Bohm effect is useful for the determination of the ordering of bright and dark excitons.

Brightening of dark excitons in magnetic flux was observed in recent photoluminescence experiments [56-59]. Fine structures, spectral diffusion, and intermittency of photoluminescence were also suggested to be related to dark excitons $[60,61]$. The exciton fine structure has been discussed also in different methods including first-principles [49,62-64]. The Aharonov-Bohm effect on the dark and bright excitons was also suggested in Ref. [54].

7 Exciton absorption for cross-polarization The inter-band absorption for perpendicular polarization is suppressed considerably because of strong depolarization effect $[11,12]$. However, a peak appears when the strong exciton effect is taken into account [65]. When the depolarization effect is ignored completely, the dynamical conductivity giving the absorption spectrum is given by conductivity $\sigma_{x x}^{l}(\omega)$ with $l= \pm 1$, describing current induced by an electric field with wave number $2 \pi l / L$ along the circumference direction.

The depolarization effect is included in a self-consistent manner by considering the electric field corresponding to the induced polarization $[11,12]$. Then, the effective dynamical conductivity becomes

$$
\tilde{\sigma}_{x x}^{l}(\omega)=\frac{\sigma_{x x}^{l}(\omega)}{\varepsilon_{x x}^{l}(\omega)}, \quad \varepsilon_{x x}^{l}(\omega)=1+\frac{4 \pi^{2} \mathrm{i}|l|}{\kappa L \omega} \sigma_{x x}^{l}(\omega) .
$$

The absorption is proportional to the real part of $\tilde{\sigma}_{x x}(\omega)=$ $(1 / 2)\left[\tilde{\sigma}_{x x}^{+1}(\omega)+\tilde{\sigma}_{x x}^{-1}(\omega)\right]$. This shows that the excitation energy is given by a zero point of the dielectric function $\varepsilon_{x x}^{ \pm 1}(\omega)$. Actually, we have $\sigma_{x x}^{+1}(\omega)=\sigma_{x x}^{-1}(\omega)$ within the present effective-mass scheme and therefore $\varepsilon_{x x}^{+1}(\omega)=\varepsilon_{x x}^{-1}$ $(\omega)$. In the presence of a slight asymmetry between the conduction and valence bands, we have $\sigma_{x x}^{+1}(\omega) \neq \sigma_{x x}^{-1}(\omega)$ and therefore the absorption peak splits into two.

Figure 11 shows some examples of calculated absorption spectra. The dashed lines change into the solid lines by the depolarization effect. The absorption intensity is reduced by the depolarization effect, but the exciton mani-

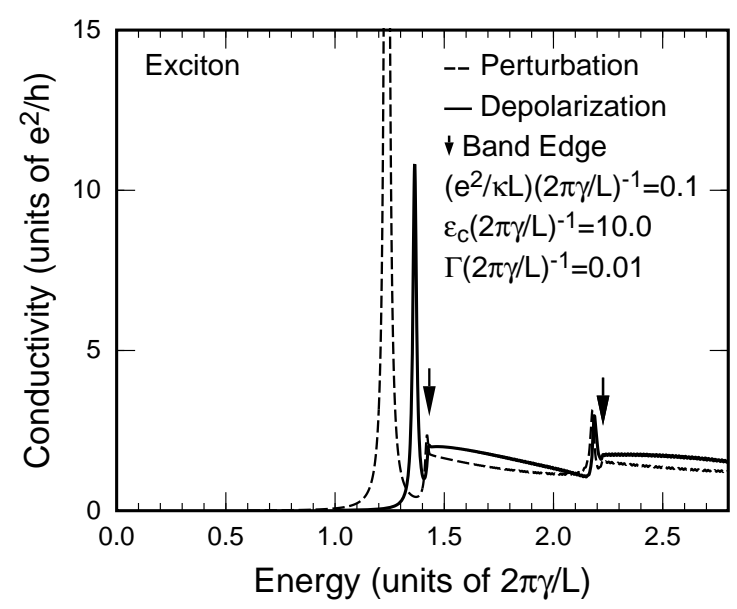

Figure 11 Examples of calculated absorption spectra for light polarized perpendicular to the axis. The dashed lines turn into the solid lines by the depolarization effect. $\left(e^{2} / \kappa L\right)(2 \pi \gamma / L)^{-1}=$ 0.1. After Ref. [65]. 


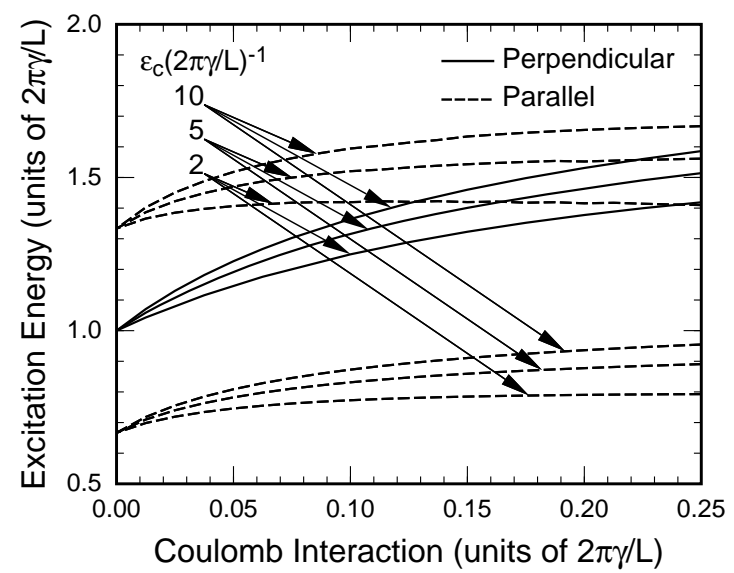

Figure 12 The exciton energies for the perpendicular (solid lines) and parallel polarization (dashed lines). The dependence is larger for the perpendicular polarization. After Ref. [65].

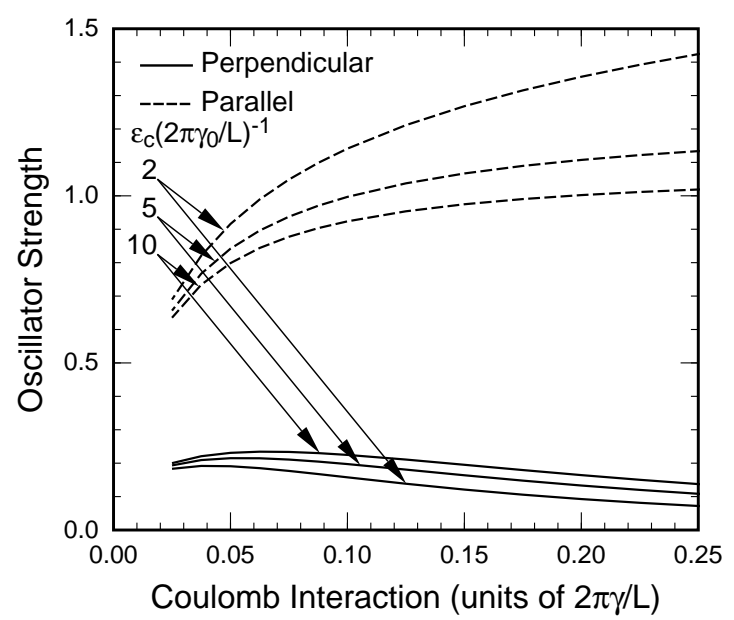

Figure 13 The oscillator strength of the exciton absorption for the perpendicular (solid lines) and parallel polarization (dashed lines). After Ref. [65].

fests itself due to its large binding energy. Figure 12 shows the exciton energies for both parallel and perpendicular polarization. In the absence of interaction, the excitation energy for the perpendicular polarization is at the middle of those of the first and second gaps in the case of the parallel polarization. For the interaction strength $\left(e^{2} / \kappa L\right)(2 \pi \gamma /$ $L)^{-1} \sim 0.15$ corresponding to actual systems, however, the exciton energy for the perpendicular polarization is closer to the second gap.

Figure 13 compares the oscillator strength of the exciton absorption between the case of the parallel and perpendicular polarization. The strength for the perpendicular polarization is about 10-20 \% of that for the parallel polarization. In spite of this weakening of the intensity, it is certainly possible to observe exciton peaks for the perpen- dicular case. It should be noted that when the symmetry between the conduction band at the $\mathrm{K}$ point and the valence band at the $\mathrm{K}^{\prime}$ point or vise versa is violated by magnetic flux, optical transitions at exciton energies without depolarization effect become weakly allowed [66]. A similar brightening of dark excitons is also possible by higherorder $\mathbf{k} \cdot \mathbf{p}$ terms.

Photoluminescence spectra were decomposed into those associated with absorption of parallel and perpendicular light [67]. The obtained spectra for the perpendicular polarization showed that a peak with intensity, about an orderof-magnitude smaller than that for the parallel polarization, appears at a position closer to that of the second lowest peak for the parallel polarization. This is consistent with the theoretical prediction. The exciton peaks for the perpendicular polarization were also observed in a single nanotube [68]. Theoretical discussion was made in very thin nanotubes $[25,62]$.

8 Summary A brief review has been given on the electronic and optical properties of carbon nanotubes. The topics have included an effective-mass description of the electronic states, optical absorption and excitons, two-photon absorption, excitons in metallic nanotubes, their fine structure and the Aharonov-Bohm effect, and exciton absorption for perpendicular polarization.

Acknowledgements This work was supported in part by Grants-in-Aid for Scientific Research and Scientific Research on Priority Area "Carbon Nanotube Nano-Electronics" from the Ministry of Education, Culture, Sports, Science and Technology, Japan.

\section{References}

[1] H. Ajiki and T. Ando, J. Phys. Soc. Jpn. 62, 1255 (1993).

[2] T. Ando, J. Phys. Soc. Jpn. 74, 777 (2005) and references cited therein.

[3] H. Ajiki and T. Ando, J. Phys. Soc. Jpn. 62, 2470 (1993); J. Phys. Soc. Jpn. 63, 4267 (1994) (Erratum).

[4] P. R. Wallace, Phys. Rev. 71, 622 (1947).

[5] J. C. Slonczewski and P. R. Weiss, Phys. Rev. 109, 272 (1958).

[6] T. Ando, J. Phys. Soc. Jpn. 69, 1757 (2000).

[7] C. L. Kane and E. J. Mele, Phys. Rev. Lett. 78, 1932 (1997).

[8] H. Suzuura and T. Ando, Phys. Rev. B 65, 235412 (2002).

[9] L. Yang, M. P. Anantram, J. Han, and J. P. Lu, Phys. Rev. B 60, 13874 (1999).

[10] L. Yang and J. Han, Phys. Rev. Lett. 85, 154 (2000).

[11] H. Ajiki and T. Ando, Physica B 201, 349 (1994).

[12] H. Ajiki and T. Ando, Jpn. J. Appl. Phys. Suppl. 34-1, 107 (1995).

[13] T. Ando, J. Phys. Soc. Jpn. 66, 1066 (1997).

[14] E. A. Taft and H. R. Philipp, Phys. Rev. 138, A197 (1965).

[15] H. Sakai, H. Suzuura, and T. Ando, J. Phys. Soc. Jpn. 72, 1698 (2003).

[16] H. Sakai, H. Suzuura, and T. Ando, Physica E 22, 704 (2004). 
[17] L. Hedin: Phys. Rev. 139, A796 (1965); A. W. Overhauser: Phys. Rev. B 3, 1888 (1971); L. Hedin and S. Lundqvist: Solid State Physics, ed. F. Seitz, D. Turnbull and H. Ehrenreich (Academic, New York, 1969) Vol. 23, p. 1.

[18] T. Ando, J. Phys. Soc. Jpn. 73, 3351 (2004).

[19] M. Ichida, S. Mizuno, Y. Tani, Y. Saito, and A. Nakamura, J. Phys. Soc. Jpn. 68, 3131 (1999).

[20] M. Ichida, S. Mizuno, Y. Saito, H. Kataura, Y. Achiba, and A. Nakamura, Phys. Rev. B 65, 241407 (2002).

[21] S. M. Bachilo, M. S. Strano, C. Kittrell, R. H. Hauge, R. E. Smalley, and R. B. Weisman, Science 298, 2361 (2002).

[22] T. Miyake and S. Saito, Phys. Rev. B 68, 155424 (2003).

[23] C. D. Spataru, S. Ismail-Beigi, L. X. Benedict, and S. G. Louie, Phys. Rev. Lett. 92, 077402 (2004).

[24] C. D. Spataru, S. Ismail-Beigi, L. X. Benedict, and S. G. Louie, Appl. Phys. A 78, 1129 (2004).

[25] E. Chang, G. Bussi, A. Ruini, and E. Molinari, Phys. Rev. Lett. 92, 196401 (2004).

[26] R. B. Capaz, C. D. Spataru, S. Ismail-Beigi, and S. G. Louie, Phys. Rev. B 74, 121401 (2006).

[27] T. G. Pedersen, Phys. Rev. B 67, 073401 (2003).

[28] V. Perebeinos, J. Tersoff, and Ph. Avouris, Phys. Rev. Lett. 92, 257402 (2004).

[29] Vl. A. Margulis and T. A. Sizikova, Physica B 245, 173 (1998).

[30] Vl. A. Margulis and E. A. Gaiduk, J. Opt. A: Pure Appl. Opt. 3, 267 (2001)

[31] E. J. Mele, P. Kral, and D. Tomanek, Phys. Rev. B 61, 7669 (2000).

[32] W.-D. Cheng, D.-S. Wu, X.-D. Li, Y.-Z. Lan, H. Zhang, D.-G. Chen, Y.-J. Gong, Y.-C. Zhang, F.-F. Li, J. Shen, and Z.-G. Kan, Phys. Rev. B 70, 155401 (2004).

[33] F. Wang, G. Dukovic, L. E. Brus, and T. F. Heinz, Science 308, 838 (2005).

[34] J. Maultzsch, R. Pomraenke, S. Reich, E. Chang, D. Prezzi, A. Ruini, E. Molinari, M. S. Strano, C. Thomsen, and C. Lienau, Phys. Rev. B 72, 241402 (2005).

[35] G. Dukovic, F. Wang, D. Song, M. Y. Sfeir, T. F. Heinz, and L. E. Brus, Nano Lett. 5, 2314 (2005).

[36] S. Uryu, H. Ajiki, and T. Ando, Phys. Rev. B (submitted for publication)

[37] M. J. O'Connell, S. M. Bachilo, C. B. Huffman, V. C. Moore, M. S. Strano, E. H. Haroz, K. L. Rialon, P. J. Boul, W. H. Noon, C. Kittrell, J. Ma, R. H. Hauge, R. B. Weisman, and R. E. Smalley, Science 297, 593 (2002).

[38] H. Kataura, Y. Kumazawa, Y. Maniwa, I. Umezu, S. Suzuki, Y. Ohtsuka and Y. Achiba, Synth. Met. 103, 2555 (1999).

[39] M. S. Strano, S. K. Doorn, E. H. Haroz, C. Kittrell, R. H. Hauge, and R. E. Smalley, Nano Lett. 3, 1091 (2003).

[40] C. Fantini, A. Jorio, M. Souza, M. S. Strano, M. S. Dresselhaus, and M. A. Pimenta, Phys. Rev. Lett. 93, 147406 (2004).

[41] H. Telg, J. Maultzsch, S. Reich, F. Hennrich, and C. Thomsen, Phys. Rev. Lett. 93, 177401 (2004).

[42] M. Y. Sfeir, T. Beetz, F. Wang, L. Huang, X. M. H. Huang, M. Huang, J. Hone, S. P. O'Brien, J. A. Misewich, T.F. Heinz, L. Wu, Y. Zhu, and L. E. Brus, Science 312, 554 (2006).
[43] F. Wang, D. J. Cho, B. Kessler, J. Deslippe, P. J. Schuck, S. G. Louie, A. Zettl, T. F. Heinz, and Y. R. Shen, Phys. Rev. Lett. 99, 227401 (2007).

[44] J. Deslippe, C. D. Spataru, D. Prendergast, and S. G. Louie, Nano Lett. 7, 1626 (2007).

[45] S. Uryu and T. Ando, Phys. Rev. B 76, 115420 (2007).

[46] T. Ando, J. Phys. Soc. Jpn. 75, 024707 (2006).

[47] K. Ohno, Theoret. Chim. Acta 2, 219 (1964).

[48] I. Ohmine, M. Karplus, and K. Schulten, J. Chem. Phys. 68, 2298 (1978).

[49] V. Perebeinos, J. Tersoff, and Ph. Avouris, Nano Lett. 5, 2495 (2005).

[50] S. Zaric, G. N. Ostojic, J. Kono, J. Shaver, V. C. Moore, M. S. Strano, R. H. Hauge, R. E. Smalley, and X. Wei, Science 304, 1129 (2004)

[51] S. Zaric, G. N. Ostojic, J. Kono, J. Shaver, V. C. Moore, R. H. Hauge, R. E. Smalley, and X. Wei, Nano Lett. 4, 2219 (2004).

[52] L. J. Li and R. J. Nicholas, Internat. J. Mod. Phys. B 18, 3509 (2004).

[53] S. Zaric, G. N. Ostojic, J. Shaver, J. Kono, X. Wei, M. Furis, S. A. Crooker, O. Portugall, P. H. Frings, G. L. J. A. Rikken, V. C. Mooree, R. H. Haugee, and R. E. Smalley, Physica E 29, 469 (2005).

[54] S. Zaric, G. N. Ostojic, J. Shaver, J. Kono, O. Portugall, P. H. Frings, G. L. J. A. Rikken, M. Furis, S. A. Crooker, X. Wei, V. C. Moore, R. H. Hauge, and R. E. Smalley, Phys. Rev. Lett. 96, 016406 (2006).

[55] H. Yokoi, N. Kuroda, Y. Kim, N. Minami, S. Kazaoui, K. Uchida, and S. Takeyama, in Narrow Gap Semiconductors 2005 (Institute of Physics Conference Series Number 187) edited by J. Kono and J. Leotin, (Taylor \& Francis, London, 2006), p. 278.

[56] J. Shaver, J. Kono, S. Hansel, M. Von Ortenberg, C. Mielke, O. Portugall, R. Hauge, R. Smalley, Institute of Physics Conference Series 187, 266 (2006).

[57] I. B. Mortimer and R. J. Nicholas, Phys. Rev. Lett. 98, 027404 (2007).

[58] I. B. Mortimer, L.-J. Li, R. A. Taylor, G. L. Rikken, O. Portugall, and R. J. Nicholas, Phys. Rev. B 76, 085404 (2007).

[59] J. Shaver and J. Kono, Laser Photon. Rev. 1, 260 (2007).

[60] K. Matsuda, Y. Kanemitsu, K. Irie, T. Saiki, T. Someya, Y. Miyauchi, and S. Maruyama, Appl. Phys. Lett. 86, 123116 (2005).

[61] K. Matsuda, T. Inoue, Y. Murakami, S. Maruyama, and Y. Kanemitsu, Phys. Rev. B 77, 193405 (2008).

[62] H. Zhao and S. Mazumdar, Phys. Rev. Lett. 93, 157402 (2004).

[63] V. Perebeinos, J. Tersoff, and Ph. Avouris, Phys. Rev. Lett. 94, 086802 (2005).

[64] C. D. Spataru, S. Ismail-Beigi, R. B. Capaz, and S. G. Louie, Phys. Rev. Lett. 95, 247402 (2005).

[65] S. Uryu and T. Ando, Phys. Rev. B 74, 155411 (2006).

[66] S. Uryu and T. Ando, Phys. Rev. B 77, 205407 (2008).

[67] Y. Miyauchi, M. Oba, and S. Maruyama, Phys. Rev. B 74, 205440 (2006).

[68] J. Lefebvre and P. Finnie, Phys. Rev. Lett. 98, 167406 (2007). 